Swarthmore College

Works

10-1-1991

\title{
Scaling Behavior Of Atomic Trajectories In Confined Fluids
}

Amy Lisa Graves

Swarthmore College, abug1@swarthmore.edu

B. J. Berne

Follow this and additional works at: https://works.swarthmore.edu/fac-physics

Part of the Physics Commons

Let us know how access to these works benefits you

\section{Recommended Citation}

Amy Lisa Graves and B. J. Berne. (1991). "Scaling Behavior Of Atomic Trajectories In Confined Fluids". Physical Review A. Volume 44, Issue 8. 4953-4960. DOI: 10.1103/PhysRevA.44.4953

https://works.swarthmore.edu/fac-physics/179

This work is brought to you for free by Swarthmore College Libraries' Works. It has been accepted for inclusion in Physics \& Astronomy Faculty Works by an authorized administrator of Works. For more information, please contact myworks@swarthmore.edu. 


\title{
Scaling behavior of atomic trajectories in confined fluids
}

\author{
A. L. R. Bug \\ Department of Physics and Astronomy, Swarthmore College, Swarthmore, Pennsylvania 19081
}

B. J. Berne

Department of Chemistry, Columbia University, New York, New York 10027

(Received 5 February 1990; revised manuscript received 29 April 1991)

\begin{abstract}
We study the dependence of $D$, a quantity that has previously been associated with the fractal dimension of an atomic trajectory, on the time scale on which it is measured. Single-particle and relative trajectories are generated by molecular-dynamics simulations of Lennard-Jones atoms permanently trapped in a spherical pore. Transient trapping is studied with a generalized Langevin model of dynamics. Confinement of trajectories allows $D$ to exceed 2; in the case of permanent trapping, $D$ diverges.
\end{abstract}

PACS number(s): 05.40. $+\mathrm{j}, 61.20 . \mathrm{Ja}, 66.10 . \mathrm{Cb}$

\section{INTRODUCTION}

Recently, the trajectories of individual atoms in fluids have been analyzed with the goal of obtaining the fractal dimension [1] of the trajectory as a function of its duration. This dimension $D$ is defined in terms of the length $L$ and units of measure $\epsilon$ as

$$
L(\epsilon)=C_{0} \epsilon^{1-D},
$$

where $C_{0}$ is some constant factor. A variation of $D$ with $\epsilon$ classifies the trajectory as being a multifractal with a geometry that is self-affine (as opposed to self-similar) [2]. Studies by Powles and Quirke [3], Powles [4], Rapaport [5], and Toxvaerd [6] employed trajectories generated by molecular dynamics or Monte Carlo simulation; Brooks [7] and Toxvaerd [6,8] explored the consequences of using a generalized Langevin equation (GLE) for the dynamics of a fluid atom. These studies have produced a picture of a particle whose short-time inertial behavior and long-time diffusive behavior are bridged by an extended crossover region, in which the trajectory dimension crosses from the inertial value of 1 to the Brownian value of 2 . Fluid memory is important in determining the crossover behavior; in particular, the algebraic decay of velocity self-correlations at long times [9] produces a very slow approach to the Brownian limit [8]. Further, the Verlet parametrization [10] of the memory function was used in Ref. [7] to obtain values of $D(t)>2$ for intermediate times. Here, we investigate a disagreement in the definition of "trajectory dimension" as calculated by previous authors; we continue to study the effect of memory friction; and we present molecular-dynamics results which characterize $D$ as calculated in [7] and [8] for Lennard-Jones (LJ) atoms confined in idealized pores.

This paper is organized as follows. In Sec. II, we review the definition of $D(t)$ in terms of the velocity history of a particle. We point out that when $D$ is calculated as in Refs. [7] and [8], there is a relationship between the viscous and elastic time scales for a viscoelastic fluid and the asymptotic behavior $D(t)$ as $t \rightarrow \infty$. In Sec. III, we apply the calculation to a dense fluid in a microscopic pore, where the lack of macroscopic diffusion results in a trajectory dimension which increases without bound.

\section{TRAJECTORY LENGTH AND THE GLE}

Consider the motion of a fluid atom during a time interval of duration $\tau_{\epsilon}$. During this time, the atom travels a mean distance $\epsilon$, which is imagined to be the smallest scale at which one can resolve segments of the atomic trajectory. The expected square of the displacement of the atom in this interval is

$$
\epsilon^{2}=\left\langle\left|\mathbf{r}\left(\tau_{\epsilon}\right)-\mathbf{r}(0)\right|^{2}\right\rangle,
$$

where \langle\rangle denotes an average over atomic trajectories. It is useful, both for comparison with scattering data and for studying model systems, to write $\epsilon$ in terms of the velocity autocorrelation function (VACF), $C_{v}(t) \equiv\langle\mathbf{v}(t) \cdot \mathbf{v}(0)\rangle /\left\langle\mathbf{v}^{2}\right\rangle$, as $[7,8]$

$$
\epsilon^{2}=\frac{6 k T}{m} \int_{0}^{\tau_{\epsilon}} d t \int_{0}^{t} d t^{\prime} C_{v}\left(t^{\prime}\right)
$$

If one observes the trajectory for a time $\Upsilon$ which is large compared to $\tau_{\epsilon}$, then the path length of Eq. (1) can be rewritten as

$$
L(\epsilon)=\frac{\Upsilon}{\tau_{\epsilon}} \epsilon=N(\epsilon) \epsilon+\operatorname{ord}(\epsilon),
$$

where $N$ is the closest integer less than $\Upsilon / \tau_{\epsilon}$, and it corresponds to the expected number of intervals of length $\epsilon$ needed to approximate the path. The correction term emphasizes the uncertainty of order unity in the number of these increments in any given path. This correction term is irrelevant if the number of path increments $N(\epsilon)$ is sufficiently large; henceforth, we will omit it.

In several recent studies which apply notions of fractal dimension to trajectories, Eq. (1) is replaced by the defining equation 


$$
D(\epsilon)=1-\frac{d \ln L(\epsilon)}{d \ln \epsilon}
$$

Equation (5), an alternative to the Richardson relation Eq. (1), turns out to be a starting point for analysis in Refs. [7], [8], and [11]. That is, if one substitutes for $L$ from the first part of Eq. (4), the simple relation

$$
D(\epsilon)=\frac{\partial \ln \tau_{\epsilon}}{\partial \ln \epsilon}
$$

results. That is,

$$
D(\epsilon)=\frac{2}{\tau_{\epsilon}} \frac{\int_{0}^{\tau_{\epsilon}} d t \int_{0}^{t} d t^{\prime} C_{v}\left(t^{\prime}\right)}{\int_{0}^{\tau_{\epsilon}} d t C_{v}(t)} .
$$

In the references mentioned above, Eq. (7) is explored for model liquids. Note, however, that Eq. (5) is not equivalent to Eq. (1) if there is a variation of $D$ with the scale, so its derivative with respect to $\ln \epsilon$ does not vanish. If one hopes to use the original Richardson expression, then in place of Eq. (7) one has

$$
D(\epsilon)=\frac{\ln \tau_{\epsilon}}{\ln \epsilon}+\frac{\text { const }}{\ln \epsilon},
$$

where the constant in Eq. (8) is $\ln C_{0} / \ln \Upsilon$.

Equations (7) and (8) produce different predictions of $D$; some thought is required to determine which value more adequately reflects the "path dimension." One must realize that in the definition Eq. (1), the requirement that $C_{0}$ is really a constant independent of $\epsilon$ is possible only if $D$ is independent of $\epsilon$. This is apparent in the following example. Consider a fixed piece of a trajectory which has the property of being ballistic on a short length scale and diffusive over long distance scales. If one begins by satisfying Eq. (1) for intervals on the small, ballistic length scale where $D=1$, then one can call $C_{0}=L_{0}$, the length of a piecewise-linear trajectory. On the other hand, suppose one starts the analysis with an arbitrarily long length scale $\sigma_{0}$ and one measures the trajectory to have a length $L_{1}$. Then the definition $C_{0}=\sigma_{0} L_{1}$ will make Eq. (1) true for $D=2$ for all ruler lengths $\epsilon>\sigma_{0}$. And, it is not true in general that these two values for $C_{0}$ are the same; if the trajectory were truly a sum of random vectors of length $\sigma_{r}$, then $L_{1}=\left(L_{0} \sigma_{r}\right)^{1 / 2}$. Thus, $C_{0}$ is a function of $L_{0}$ and of $\epsilon$, and exhibits a crossover with $\epsilon$ in this example.

This $\epsilon$ dependence implies that if one tries to calculate $D$ from the slope of $\ln L$ vs $\ln \epsilon$, as has been done previously, one will find a variation in $D$ with the ballistic length (or alternatively, with trajectory duration) for fixed $\epsilon$, even if the trajectory is long enough for the motion to be purely diffusive at the largest length scale. Consider a piece of a trajectory with ballistic length $L_{0}$. Then from Eq. (1),

$$
\frac{\partial L(\epsilon)}{\partial \ln \epsilon}=\frac{\partial C_{0}(\epsilon)}{\partial \ln \epsilon}+\frac{\partial[1-D(\epsilon)] \ln \epsilon}{\partial \ln \epsilon} .
$$

If one omits the term in $\partial D / \partial \ln \epsilon$, the second terms reduces to $1-D$. If one also omits the first term, one ar- rives at Eq. (5), and would associate $1-D$ with the slope on a plot of $\ln L$ vs $\ln \epsilon$. However, the existence of this term alters this slope. We can find the direction of this alteration for the simple ballistic-to-diffusive example which is discussed above. In this case, $C_{0}$ decreases as $\epsilon$ grows, so this correction term is negative. Since $C_{0}$ grows as $L_{0}$ grows, $D$ will appear larger and larger for trajectories with longer and longer ballistic lengths (or alternatively, longer durations). In Ref. [4], just such a behavior was found in simulated data for a $\mathrm{LJ}$ fluid, and in Ref. [12] for Monte Carlo trajectories with and without random scatterers. Trajectories agreed on the short-time ballistic exponent $D=1$, but predicted different exponents $D(\epsilon)$, evaluated at identical intermediate length scales. Some workers argue that trajectories which show stable values of $D<2$ for large $\epsilon$ are not long enough. We suggest that even trajectories which are long enough to exhibit true diffusive behavior at the largest scale may exhibit different values of $D$ at intermediate scales, if the slope of $\ln L$ is interpreted as $1-D$. For example, Erpenbeck and Cohen [11] perform a careful study of hard spheres and deduce $D$ in this way [as well as by direct calculation of the VACF for use in Eq. (7)]. They find that while $D$ seems to saturate well below 2 for a trajectory of fixed duration $\Upsilon$ of about 2000 mean free times, trajectories of various durations disagree on $D(\epsilon)$. This allows them to perform an extrapolation of $D(\epsilon)$ to a trajectory of infinite duration. The extrapolation shows the hopedfor behavior: a continued rise toward the Brownian value of $D=2$.

The discrepancy between using Eqs. (7) and (8) to evaluate $D$ may explain the fact that Ref. [4], in which the latter definition is implicity used, finds that $D$ crosses over logarithmically slowly to the Brownian value of 2 , while Ref. [7], which uses the former definition, finds a qualitatively different result - a much swifter crossover. Though the definition of a fractal dimension which is a function of time seems to demand the use of Eq. (8), a dependence of $D$ on length and time scales exponentially larger than $\epsilon$ and $\tau_{\epsilon}$ is not desirable. Nor is the resulting logarithmic crossover to $D=2$ desirable. Both of these are artifacts of the assumed constancy of $C_{0}$; if one had some $a$ priori model for the functional form of either $D$ or $C_{0}$, one could fit the data for the other. We do not have such a model for these functions in the crossover region. In this work, we will evaluate the consequences of beginning with the definition Eq. (5) and using Eq. (7) to find $D$ from the VACF. We note that it is incorrect to interpret $D$ as a Richardson fractal dimension for the reasons discussed above. Nevertheless, the two definitions agree when $D$ takes on a constant value, for then Eq. (1) may be differentiated to yield Eq. (5) directly. Further, our choice turns out to produce a sensitive measure of the degree of confinement of trajectories. Henceforth in this work, we will call $D$ the path or trajectory exponent and will study its behavior for model systems.

As a function of time, $D$ has the correct asymptote: $D\left(\tau_{\epsilon}\right) \rightarrow 1$ as $\tau_{\epsilon} \rightarrow 0$. [We retain the notation $D($ ) for the path exponent, though now the time scale, rather than the length scale $\epsilon$, is the argument.] For short times, the path exponent rises quadratically with time as [13] 


$$
D\left(\tau_{\epsilon}\right) \approx 1+\frac{1}{12} K_{1} \tau_{\epsilon}^{2},
$$

where $K_{1}$ is the first Mori coefficient; it has the physical interpretation

$$
K_{1}=\frac{\left\langle\mathbf{F}^{2}\right\rangle}{m^{2}\left\langle\mathbf{v}^{2}\right\rangle},
$$

where $\mathbf{F}$ is the force on the atom.

To find the long-time behavior of $D$, let

$$
\mathcal{T}(t) \equiv \int_{0}^{t} d t^{\prime} C_{v}\left(t^{\prime}\right)
$$

In the infinite time limit, $\mathcal{T}$ approaches the correlation time for the VACF, $\tau_{c}$, and is proportional to the diffusion constant. Consider $D$ at times $\tau_{\epsilon}$ which are very long compared to this correlation time:

$$
\tau_{\epsilon} \gg \tau \gg \tau_{c}
$$

where $\tau$ is some intermediate time scale. We make the ansatz in three dimensions:

$$
\mathcal{T}(\tau)=\tau_{c}+\text { ord }\left[\frac{1}{\sqrt{\tau}}\right)
$$

The prefactor of the term of order $\tau^{-1 / 2}$ can be quite small; this term will be neglected temporarily and discussed at the end of this section. Then from Eq. (7),

$$
D\left(\tau_{\epsilon}\right)=2\left[1+\frac{1}{\tau_{\epsilon}}\left[\int_{0}^{\tau} d t \frac{\mathcal{T}(t)}{\tau_{c}}-\tau\right]\right] .
$$

The time $\tau$ of Eq. (12) is arbitrary, so long as it obeys the bounds above, so that we must have

$$
\int_{0}^{\tau} d t \frac{\mathcal{T}(t)}{\tau_{c}}=\tau+G
$$

where $G$ is a constant. (One expects that there are corrections to $G$ which are higher order in $\tau^{-1}$.) The sign of $G$ determines whether $D\left(\tau_{\epsilon}\right)=2$ is approached from above or below.

Consider an atom whose motion may be modeled by a GLE [14]:

$$
m \ddot{\mathbf{v}}=-m \int_{0}^{t} d t^{\prime} \mathbf{v}\left(t-t^{\prime}\right) M\left(t^{\prime}\right)+F\left(t^{\prime}\right),
$$

where $M$ and $F$ are the memory function and random force, respectively; they are related through the second fluctuation dissipation theorem. Equation (14), together with a lack of correlation between the random force and the velocity, implies that $C_{v}$ and $M$ are related by the Volterra equation, whose Laplace transform is

$$
1-s \widetilde{C}_{v}(s)=\widetilde{M}(s) \widetilde{C}_{v}(s),
$$

where $\sim$ represents the operation of Laplace transformation. Equations (13) and (15) imply that

$$
\widetilde{G}(s)+\frac{1}{s^{2}}\left[\frac{\widetilde{M}(0)}{s+\widetilde{M}(s)}-1\right] .
$$

Equation (16) may be expanded in $s$ to find the asymptotic behavior of the path exponent at large times. That is, suppose that the memory function has the small $s$ behavior

$$
\widetilde{M}(s)=C_{1}-C_{2} s+\cdots,
$$

where one can show that it is a sufficient condition for $C_{1}, C_{2}>0$ if $M(t)$ is positive definite. This will be the case if the random forces $F$ shown no negative time correlations, which is a standard case in the theory of liquids [14]. Then to lowest order in $s$

$$
\widetilde{G}(s)=\frac{1}{C_{1} s}\left(C_{2}-1\right)
$$

so that

$$
G=\frac{C_{2}-1}{C_{1}}
$$

Thus, Eq. (12) becomes

$$
D\left(\tau_{\epsilon}\right)=2\left(1+\frac{C_{2}-1}{\tau_{\epsilon} C_{1}}\right) .
$$

The Brownian value of $D=2$ is approached with a correction term varying inversely with time, and from above or below if $C_{2}$ is greater or less than unity, respectively.

As a specific case, consider a viscoelastic medium modeled with the memory function

$$
M(t)=\frac{1}{\alpha} e^{-t / \tau_{c}} .
$$

The Fourier transform of this function gives a frequency-dependent shear viscosity whose form was originally studied by Maxwell [15]. $\alpha^{-1}$ is the highfrequency rigidity modulus of the fluid. On time scales very much less than $\tau_{c}$, the fluid behaves as a rigid solid; on time scales very much greater than this value, the response of the fluid is viscous. For this model, Eq. (19) predicts that at long times

$$
D\left(\tau_{\epsilon}\right) \cong 2\left[1+\frac{\tau_{c}}{\tau_{\epsilon}}\left[1-\frac{\alpha}{\tau_{c}^{2}}\right]\right] .
$$

Thus there are two cases. If $\alpha>\tau_{c}^{2}$, the trajectory exponent approaches the Brownian limit from below. This is the case of weak solvent memory, in which the rigidity modulus is less than the square of the relaxation frequency of the medium. If $\alpha<\tau_{c}^{2}$, the trajectory exponent relaxes to $D=2$ from above. In this case, the solvent memory is strong; the time scale set by the rigidity is less than the relaxation time for this model solid. Figure 1 shows $D(\tau)$ for a model fluid with memory function given by Eq. (20) for these two cases. The monotonic increase of $D \rightarrow 2$ in the former case contrasts with the oscillation of $D$ to a value near 3 in the latter. The small ratio of the elastic to viscous time scales $\left(\tau_{c} / \sqrt{\alpha}=\sqrt{2}\right)$ insures that oscillations in $D$ are quickly damped; the particle will not be trapped for long times near its initial position. The behavior $D>2$ was noted in Ref. [7] and associated with long-lived memory effects.

In some systems, such as fluids of repulsive spheres 


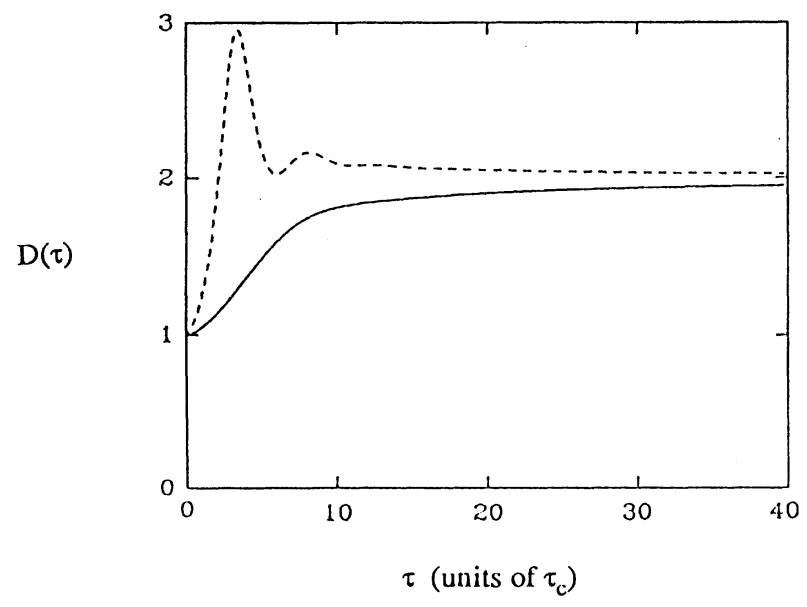

FIG. 1. $D(t)$ for particles with an exponential memory kernel; the elastic time scale $\tau_{c}$ is unity.,$- \alpha=2.0 ;---$, $\alpha=0.5$.

[16] or LJ fluids at certain state points [17], it is possible to observe a slowly decaying algebraic tail in the VACF which results form the slow decay of hydrodynamic modes in the fluid [18]. Whether or not this behavior will appear depends on the ratio of the diffusion constant $\beta$ to the kinematic viscosity $\beta_{T}$. If the ratio is large, then diffusive shear excitations will act back only weakly on a diffusing particle, and this tail will be suppressed [19]. Further, a viscoelastic fluid, such as a simple liquid near its triple point, may fail to exhibit the algebraic tail (or rather, it makes a negligible contribution to the form of the VACF, because it has been shifted to very long times) due to the frequency dependence of the viscosity [20]. In such cases, we expect the analysis above to hold. Otherwise, a correction of ord $\left(\tau^{-1 / 2}\right)$ may dominate the term $G$ in Eq. (13). For example, should the VACF exhibit an algebraic tail without caging, such as is the case at with hard spheres at low density $[16,21]$, then Toxvaerd's prediction [Ref. [8], Eq. (10)] will hold, and

$$
D \cong 2\left[1-\frac{k T}{6 \rho m}\left[\pi\left(\beta+\beta_{T}\right)\right]^{3 / 2} \tau_{\epsilon}^{-1 / 2}\right]
$$

\section{LJ FLUID IN A SPHERICAL PORE}

Homogeneous fluids at high densities or low temperatures exhibit interesting dynamical relaxation properties which may be attributed to temporary caging of fluid molecules by their near neighbors. The dynamical behavior of fluids which are permanently caged, for example, in the interiors of spherical micelles or within small chambers of a microporous solid such as a zeolite [22], is also interesting, and of technological relevance. We have studied the dynamics of a neat fluid of LJ atoms in an idealized spherical pore via molecular dynamics. Details of this simulation are reported elsewhere [23]; briefly, atoms are confined withing a spherical region of space of radius $R_{c}$ by a static, spherically symmetric wall potential. This wall potential is imagined to arise from a $\mathrm{LJ}$ interaction integrated over a spherical shell of $\mathrm{LJ}$ atoms. Thus, there is an attractive region (width $0.25 \sigma$, depth 10 $\mathrm{K})$ which is too shallow to affect the dynamics of the particles at the temperatures $(300 \mathrm{~K})$ studied. Most important is the repulsive part of the potential which varies, to leading order, as $\left(R_{c}-r\right)^{-10}$. The interparticle interaction is considered to be pairwise additive, of LJ 6-12 form, and the strength and range of the interaction are chosen to correspond to xenon: $\epsilon=229 \mathrm{~K}, \sigma=4.05 \AA$ [24]. The dynamical evolution of the system is simulated by integrating Newton's equations of motion using a Velocity Verlet [25] algorithm with a time step on the order of $0.0055 \mathrm{ps}$, which was small enough to conserve energy to within a few percent for the duration of the study. Since the number of particles is quite small, the uncertainty in the kinetic temperature $T$ is dominated by variation in $T(E)$ from realization to realization, rather than a drift in total energy $E$. For the simulation, the atomic mass was chosen to be 129 proton masses, to correspond to the isotope of xenon which is a popular NMR probe of zeolitic solids.

A set of three pore radii $R_{c}=6.98,8.60$, and $11.41 \AA$ were studied for $N=10,20$, and 50 xenon atoms. These numbers were chosen so that the average reduced density $\rho^{*}=N \sigma^{3} / V_{\text {eff }}$ remains roughly constant, $\rho^{*} \approx 0.61 \pm 0.1$. $V_{\text {eff }}$ is defined as $(4 \pi / 3) R_{\text {eff }}^{3}$ where $R_{\text {eff }}$ can be defined from the static density profile as the radial position at which the density falls, say, to $10 \%$ of its value. Plots of the density as a function of radial position (Ref. [23]) indicate that at the temperature of approximately $321 \mathrm{~K}$ $\left(T^{*} \approx 1.4\right), R_{\text {eff }} \approx 6.40,8.06$, and $10.90 \pm 0.05 \AA$ making $\rho^{*} \approx 0.605,0.605,0.612$, respectively, for the three values of $N$ studied. Figure 2(a) shows the mean-squared displacement of an atom in the cavity as a function of time $\left\langle|\mathbf{r}(t)-\mathbf{r}(0)|^{2}\right\rangle$ as in Eq. (2) of Sec. II. This quantity gives an alternate measurement of the effective cavity radius. That is, the displacement, averaged over all starting positions in the cavity, can be very well fit at long times by the form

$$
\left\langle|\mathbf{r}(t)-\mathbf{r}(0)|^{2}\right\rangle \cong a R_{\mathrm{eff}}^{2}-b R_{\mathrm{eff}}^{2} e^{-\gamma t} .
$$

This form is suggested by the solution to the diffusion equation in a spherical cavity at long times [26]. The quantity $a R_{\text {eff }}^{2}$ is simply $\left\langle 2 r^{2}\right\rangle$, where this static average over $r$ is weighted by the observed radial density. Were all positions in the cavity equally likely to be occupied by particles, the constant $a$ would be precisely $\frac{6}{5}$. Though we know that this is not the case (the radial density shows distinct oscillations typical of moderately dense fluids near hard walls) substituting $\frac{6}{5}$ for $a$ and performing a least-squares fit produces values of $R_{\text {eff }}$ which are quite consistent with the static estimates: $R_{\text {eff }}=6.48$, 8.01 , and $10.80 \AA$, respectively. Figure 2(b) shows the VACF for the three systems; the first zero crossing occurs roughly at the time that a typical particle has suffered a single collision with the cavity wall. The re- 
gion of negative correlation arises from the coherent backscattering of fluid atoms from the permanent cage.

From either the data of either Figs. 2(a) or 2(b) (the former can be obtained by integrating the latter twice with respect to time) one can calculate $D(t)$ as defined in Eq. (7) of Sec. II. The numerical integration of the VACF was performed using Simpson's rule using data taken every $0.055 \mathrm{ps}$ (every ten MD times steps). This trajectory exponent is shown for the $N=10$ system in Fig. 3(a), along with the trajectory exponent of a model system which obeys a simple Langevin equation with correlation (braking) time $\tau_{c}=0.6 \mathrm{ps}$. This time was chosen as a good fit to the short-time behavior of $D(t)$ for the cavity system, and we associate $\tau_{c}$ with the mean free time for a fluid atom. A notable feature of these data is the $D(t)$ for the model system never levels off at the Brownian value of 2 . However, to decide whether the
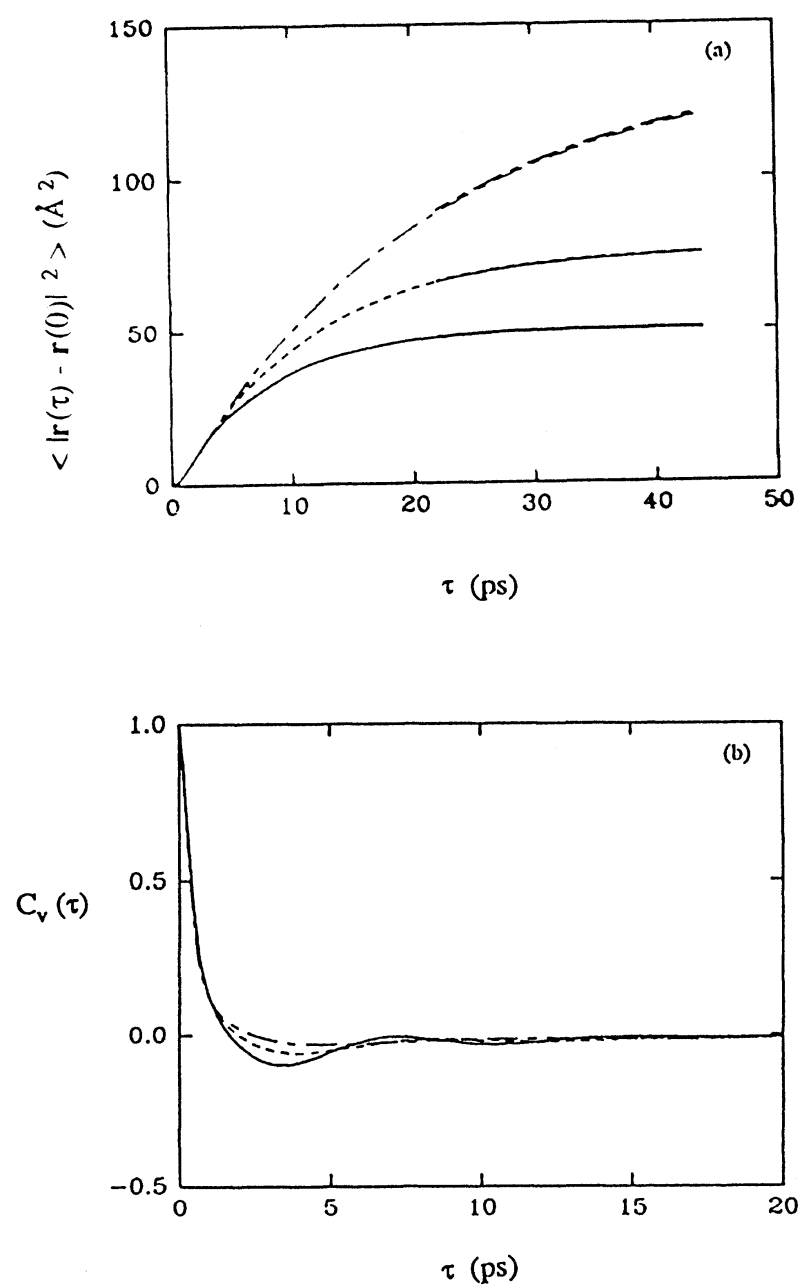

FIG. 2. LJ particles in a spherical cavity. $\rho^{*} \approx 0.61$, $T^{*} \approx 1.4$. $-N=10 ;---, N=20 ;-\cdot-\cdot-\cdot, N=50$. (a) $\left\langle|\mathbf{r}(t)-\mathbf{r}(0)|^{2}\right\rangle$. Thick lines are fit to the functional form of Eq. (22), which determines $a, b$, and $\gamma$ given $R_{\text {eff }}$ from the radial density profile or determines $R_{\text {eff }}$ and $\gamma$ given $a$ and $b$ from the assumed profile. (b) Normalized VACF.
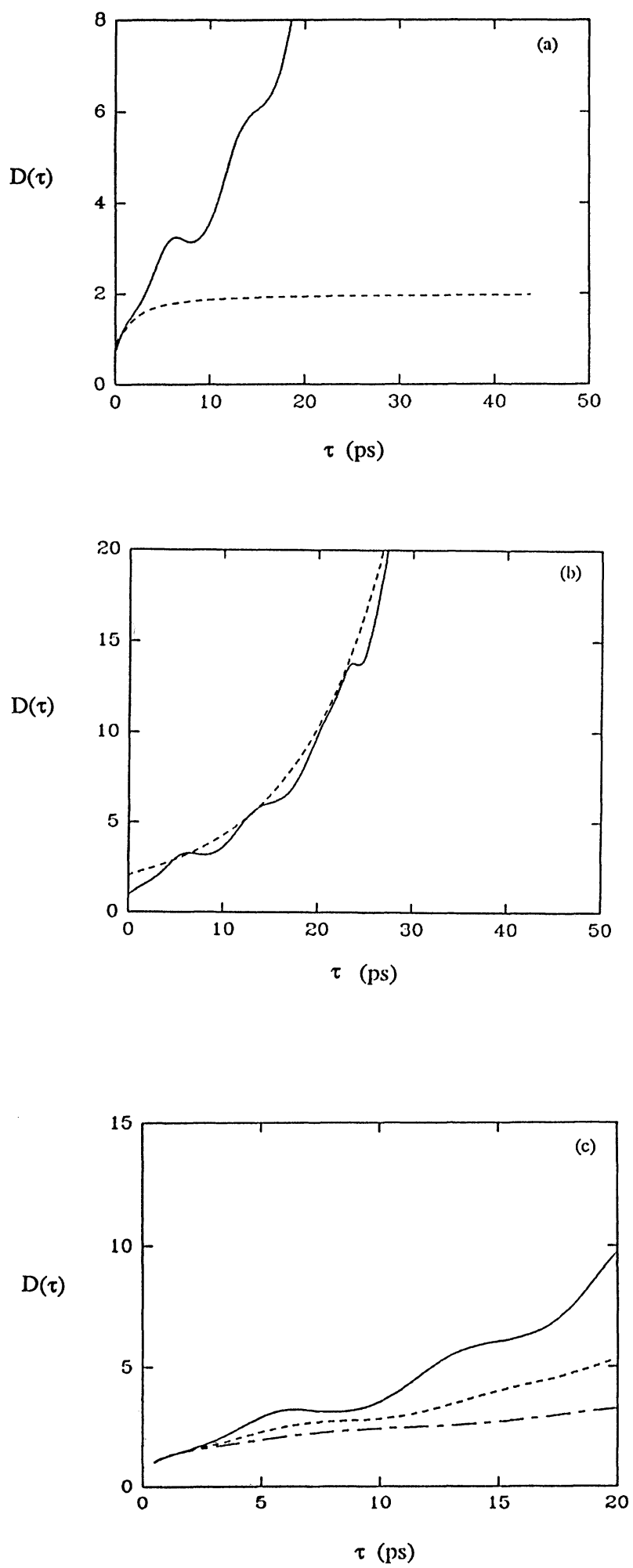

FIG. 3. Fractal path dimension. (a) _- data of Fig. 2, $N=10$; -- , Langevin model, $\tau_{c}=0.6$ ps. (b),$- N=10$, date of Fig. 2 compared with --- ; the solution to the diffusion equation in a spherical cavity with a hard wall at the effective radius of the simulated cavity. The diffusion constant is $\beta=1.3$. (c) Data of Fig. 2 . 
motion of the caged particles is diffusive at long-time scales, the relevant comparison is one with $D$ as calculated from the trajectory of an ideal diffuser in a spherical cavity. The notion of such "hindered diffussion" is often invoked for fluids in microscopic pore spaces, and one would like to understand whether fluid dynamics conforms to such a model. In this context, if the mean free path is greater than the pore diameter, the process is one of "Knudsen" diffusion [27]. Our simulated fluid, which is representative of a moderately dense gas not far from the triple point density $\left(\rho / \rho_{\text {triple }} \approx 0.72\right)$ with a temperature near the critical temperature $\left(T / T_{c} \approx 1.08\right)$ [28], displays mean times for collisions with the cavity walls on the order of 2 to $3 \mathrm{ps}$. Traditionally, this system might be considered well on the way to crossing over from Knudsen to normal diffusion, albeit in a hindering geometry, so comparison with the ideal diffuser is relevant. A second reason to ascertain whether dynamics is truly diffusive arises on the study of bimolecular reactions which are "diffusion limited" and occur in a constraining pore space [29]. These models assume that reagents diffuse within, say, the center of a micelle; a diffusive trajectory culminates in a bimolecular reaction. Such models, which predict rate constants and branching ratios, are predicated on the notion that reagents execute diffusive motion on a time scale which is much shorter than the typical time it takes the reagent to explore a micellar diameter.

In Fig. 3(b), we compare $D$ for a MD trajectory with the exact result [30] for a diffuser in a cavity with perfectly reflecting walls of radius $R_{\text {eff }}$. The diffusion constant $\beta \approx 1.3$ was chosen by fitting the MD data to the asymptotic form, Eq. (22). For the MD data, $D(t)$ diverges as $t \rightarrow \infty$ with a slight oscillatory character. These oscillations may be compared with those that appear at short times for the transiently trapped system of Fig. 1. If Eq. (7) is applied to the asymptotic form, Eq. (22), one sees

$$
D(t) \propto \frac{1}{t} e^{\gamma t} .
$$

Equation (23), which is the long-time scaling behavior of $D$ for a true diffuser in a cavity with perfectly reflecting spherical walls, has no oscillations, nor does the solution for $D$ at general times [30]. The oscillations, which are present at a very low amplitude in the VACF of Fig. 2(b), must be attributed to the ballistic nature of the atoms. Note, however, that $\left\langle|\mathbf{r}(t)-\mathbf{r}(0)|^{2}\right\rangle$ itself is monotonic; the ringing occurs in higher time derivatives. The simulated and calculated curves agree in an approximate manner, though at short times there is the inevitable discrepancy between ballistic and diffusive exponents. Figure 3(c) shows $D(t)$ for systems with sizes $N=10,20$, and 50 for times up to 20 ps. Data for times below 1 ps are not shown; because $D$ is found by numerical integration with a time step of around $0.05 \mathrm{ps,} \mathrm{one} \mathrm{might} \mathrm{not} \mathrm{ob-}$ serve the correct time dependence for integrals over only a few of these elementary time steps.

The divergence of $D(t)$ at long times, like the result $D>2$ for transiently caged fluids, is reasonable for a bounded trajectory. We have emphasized that $D$ of Eq. (23) is not a fractal dimension. If we had, instead, adopt- ed Eq. (8) for $D(t)$, we would have also found a divergence:

$$
D(t) \propto \ln (t) .
$$

Intuitively, one thinks of a fractal path as one with an infinite hierarchy of features on an infinite hierarchy of length scales. A way to describe such a path is to assert that as $\epsilon$ grows, one finds fewer and fewer path segments of scale $\epsilon$. The number $N(\epsilon)$ [Eq. (4)] counts these segments. Thus, for a fixed trajectory of exponent $D$, if one expands the scale at which one measures the trajectory by $\alpha$, the number of path segments of that length should decrease by a factor of $\alpha^{D-1}$. For a trapped system, there are no segments of length greater than the effective diameter of the trap. As the trajectory is measured on longer and longer time scales, corresponding to longer and longer length scales (bounded above by this trapping length), fewer and fewer path segments which span these long length scales can be found. For this reason, whether
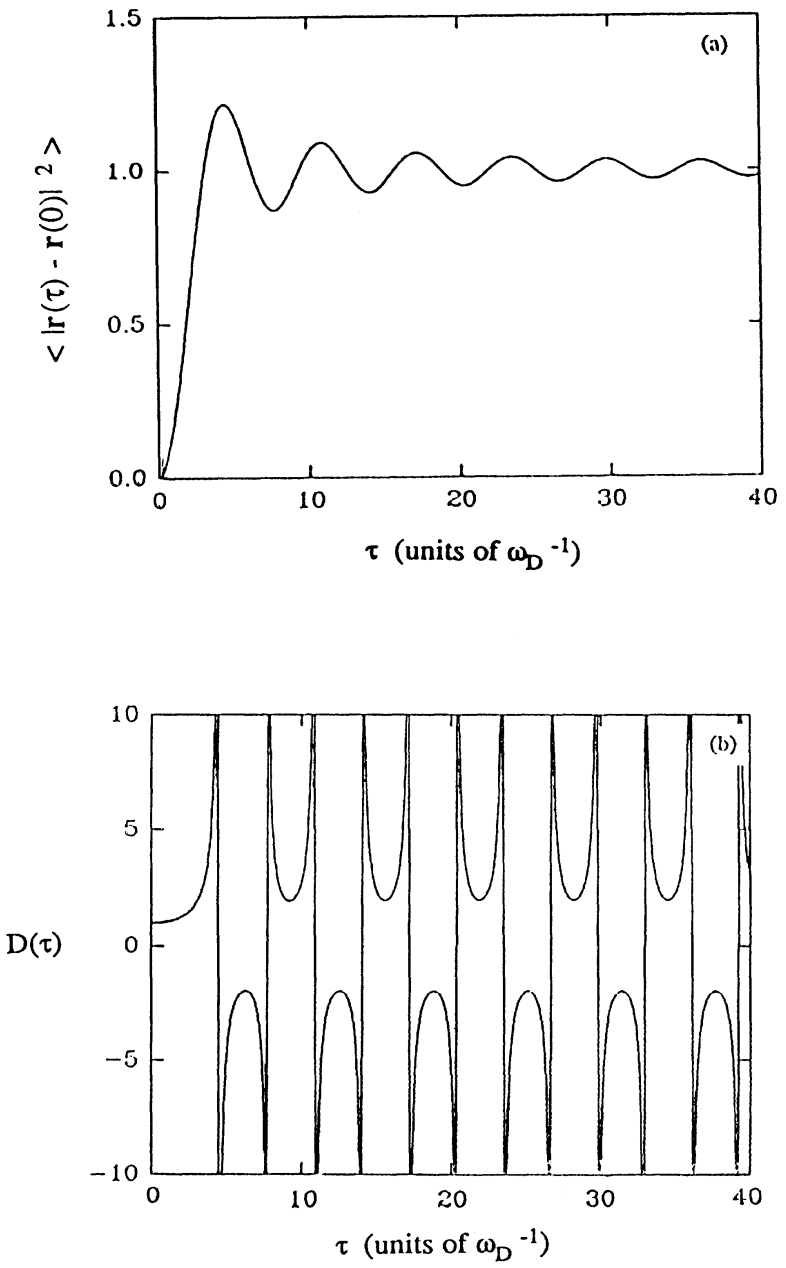

FIG. 4. Vinyard model of displacement of an atom in the Debye solid. (a) $\left\langle|\mathbf{r}(t)-\mathbf{r}(0)|^{2}\right\rangle$, in units where $18 k T / m \omega_{D}=1$. (b) Fractal path dimension. 
we adopt Eqs. (7) or (8), the exponent $D-1$ reflects this scarcity of path segments and diverges as time increases.

As an example of a trajectory with a more pronounced ballistic ringing behavior, one can examine an atomic trajectory in a solid. One finds that for Vinyard's [31] model of the displacement of an atom in a harmonic cubic Debye lattice,

$$
\left\langle|\mathbf{r}(t)-\mathbf{r}(0)|^{2}\right\rangle=\frac{18 k T}{m \omega_{D}}\left(1-\frac{\sin \omega_{D} t}{\omega_{D} t}\right),
$$

the path exponent is

$$
D(t)=2 \frac{1-\left(\sin \omega_{D} t\right) /\left(\omega_{D} t\right)}{\left(\sin \omega_{D} t\right) /\left(\omega_{D} t\right)-\cos \omega_{D} t} .
$$

Equation (26) indicates that $D$ is unity for small times (ballistic limit) and that at later times it oscillates with a frequency which approaches the Debye frequency $\omega_{D}$.
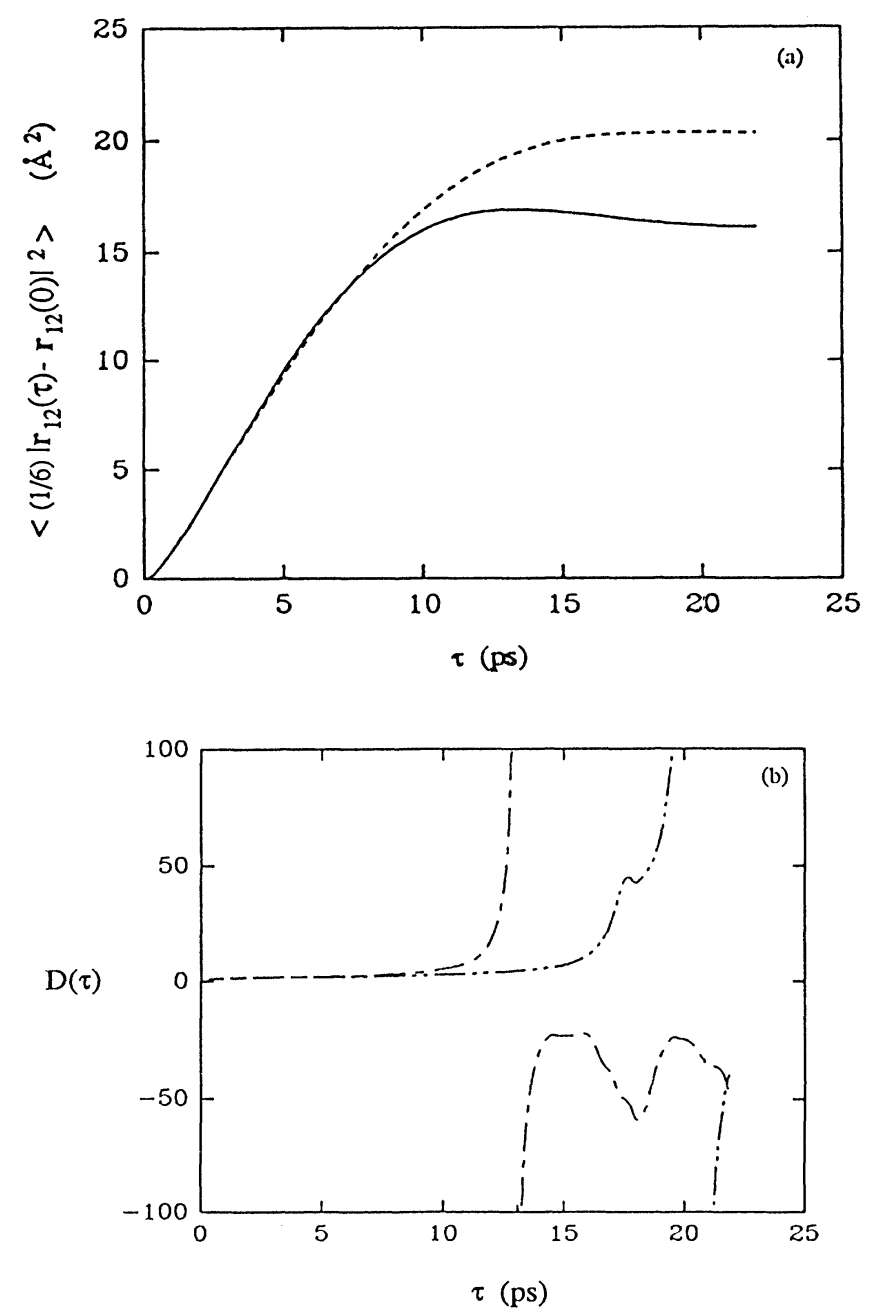

FIG. 5. LJ particles in a spherical cavity. (a) $\left\langle\frac{1}{6}\left|\mathbf{r}_{12}(t)-\mathbf{r}_{12}(0)\right|^{2}\right\rangle$. The parameters are $\rho^{*} \approx 0.8, T^{*} \approx 1.5$. , $N=10$;,$--- N=15$. (b) Fractal path dimension for relative coordinate. $-\cdot-\cdot-\cdot, N=10 ;-\cdots-\cdot, N=15$.
Figs. 4(a) and 4(b) show the mean-squared displacement and $D$, respectively. When $\left\langle|\mathbf{r}(t)-\mathbf{r}(0)|^{2}\right\rangle$ is an increasing function of time, $D$ is positive; when decreasing, $D$ is negative. These sign changes occur at places where the amplitude of $D$ has local maxima; at these maxima, $D$ is infinite. Interestingly, at long times, the magnitude of $D$ oscillates between 2 and $\infty$; the oscillator's trajectory has become déphased and shifts between free Brownian-type behavior and a behavior that reflects the constraining presence of the harmonic barrier.

A last example is relevant to diffusion-limited reactions. Figure 5(a) shows $\frac{1}{6}\left\langle\left|\mathbf{r}_{12}(t)-\mathbf{r}_{12}(0)\right|^{2}\right\rangle$ for two confined systems with $N=10$ and 15 and $\rho^{*} \approx 0.8$, where $\mathbf{r}_{12}$ is the relative displacement $\mathbf{r}_{1}-\mathbf{r}_{2}$. The average is over all initial times and initial separations of atoms 1 and 2 (and over all particle labels). The prefactor $\frac{1}{6}$ is added so that the ordinate in the figure would correspond to $t \beta_{12}$ for a uniformly diffusing system with a pair diffusion coefficient $\beta_{12}$. Figure 5 shows $D(t)$ for these systems. As in Fig. 4(b), the proximity of the pair is indicated by a low magnitude of $D$; at these times the pair coordinate is fairly free to move in time. When the typical relative separation is large, the pair coordinate is constrained by the cage and the magnitude of $D$ is large. The "derivative of a $\delta$ function" features [cf. Fig. 4(b)] are present; these are located at times when $d\left\langle\left|\mathbf{r}_{12}(t)-\mathbf{r}_{12}(0)\right|^{2}\right\rangle / d t$ vanishes. These features are present in the trajectory for pair separations, but absent in the single-particle trajectories of Fig. 3. This may occur because for pairs of $\mathbf{L J}$ particles there is a typical first meeting (second return, ... $n^{\text {th }}$ return, ...) time at which two labeled particles will approach each other and remain associated for a period of time. This information is completely contained in $\left\langle\left|\mathbf{r}_{12}(t)-\mathbf{r}_{12}(0)\right|^{2}\right\rangle$, but it appears in a subtle way. The application of Eq. (7) to produce $D(t)$ provides a clearer record of these events. The noisy behavior of $D(t)$ for the $N=10$ system after the first return time of around $15 \mathrm{ps}$ is not generic, and arises because the denominator of $\mathrm{Eq}$. (7) can propagate error in the integral of $C_{v}$ enormously if that integral is close to zero. Nevertheless, we include these data for comparison with the Vinyard model data of Fig. 4. In both cases, an expected return to an initial relative position produces a plateau in $D$.

In conclusion, we have adopted a form for the path exponent $D$ for an atom's trajectory as a function of the time scale on which it is measured, which reveals certain aspects of the trajectory in a striking manner. Unconstrained trajectories in a fluid are expected to develop the Brownian dimension of 2 , though the time scale for this can be quite long on the time scale of the relaxation of velocity correlations. Transient caging produces values of $D>2$; permanently caged atoms will have $D \rightarrow \infty$ as $t \rightarrow \infty$. Oscillations may appear in $D$ for a permanently caged atom, depending on the nature of the interatomic interactions, and the nature of any external potential. Even if the expected atomic displacements increase monotonically with time, ringing in $D(t)$ may appear, and may correspond to oscillations in the time correlations between atomic displacements and velocities. The 
development of a more consistent representation of the fractal dimension, which takes into account the variation of the length prefactor as well as the scaling exponent, is an important topic which is left open for future study.

\section{ACKNOWLEDGMENT}

We gratefully acknowledge support from the Faculty Research Support Committee of Swarthmore College.
[1] B. B. Mandelbrot, The Fractal Geometry of Nature (Freeman, San Francisco, 1982); M. Barnsley, Fractals Everywhere (Academic, New York, 1988).

[2] B. B. Mandelbrot, in Fractals in Physics, edited by L. Peitronero and E. Tosatti (North-Holland, Amsterdam, 1986).

[3] J. G. Powles and N. Quirke, Phys. Rev. Lett. 52, 1571 (1984).

[4] J. G. Powles, Phys. Lett. 107A, 403 (1985).

[5] D. C. Rapaport, Phys. Rev. Lett. 53, 1965 (1984).

[6] S. Toxvaerd, J. Chem. Phys. 82, 5658 (1985).

[7] C. L. Brooks III, Phys. Rev. A 35, 5178 (1987).

[8] S. Toxvaerd, Phys. Lett. 114A, 159 (1986).

[9] H. Mori, Prog. Theor. Phys. 33, 432 (1965); 34, 399 (1965).

[10] D. Levesque and L. Verlet, Phys. Rev. A 2, 2514 (1970).

[11] J. J. Erpenbeck and E. G. D. Cohen, J. Stat. Phys. 43, 343 (1986).

[12] M. A. Lopez-Quintela, C. Tojo, and M. C. Bujan-Nunez, Mol. Phys. 65, 1195 (1988).

[13] Note that this equation corrects a sign error in Eq. (7) of Ref. [8].

[14] B. J. Berne and G. D. Harp, Adv. Chem. Phys. 17, 63 (1970); B. J. Berne, in Physical Chemistry, an Advanced Treatise, edited by H. Eyring, D. Henderson, and W. Jost (Academic, New York, 1971), Vol. 8B, Chap 9.

[15] R. Zwanzig and R. D. Mountain, Chem. Phys. 43, 4464 (1965).

[16] B. J. Alder and T. E. Wainwright, Phys. Rev. A 1, 18 (1970), D. Levesque and W. T. Ashurst, Phys. Rev. Lett. 33, 277 (1974).

[17] P. Michels and N. J. Trappeniers, Physica A 90, 179 (1978).

[18] P. Resibois and M. DeLeener, Classical Kinetic Theory of Fluids (Wiley, New York, 1977); J. P. Boon and S. Yip, Molecular Hydrodynamics (McGraw-Hill, New York, 1980).

[19] Chr. Morkel, Chr. Gronemeyer, W. Gläser, and J. Bosse, Phys. Rev. Lett. 58, 1873 (1987).

[20] J. Bosse, W. Götze, and M. Lücke, Phys. Rev. A 20, 1603 (1979).

[21] J. R. Dorfman and E. G. D. Cohen, Phys. Rev. A 6, 776
(1972).

[22] Physics of Amphiphiles: Micelles, Vesicles and Microemulsions, V. Degiorgio and M. Corti. (North-Holland, Amsterdam, 1985); D. W. Breck, Zeolite Molecular Sieves (Wiley, New York, 1974).

[23] A. L. R. Bug, Int. J. Thermophys. 10, 469 (1989).

[24] J. O. Hirschfelder, C. F. Curtiss, and R. B. Bird, Molecular Theory of Gases and Liquids (Wiley, New York, 1954).

[25] W. C. Swope, H. C. Andersen, P. H. Berens, and K. R. Wilson, J. Chem. Phys. 76, 637 (1982).

[26] H. S. Carslaw and J. C. Jaeger, Conduction of Heat in Solids (Clarendon, Oxford, 1959).

[27] D. M. Rathven, Principles of Adsorption and Adsorption Processes (Wiley, New York, 1984), Chap. 5.

[28] The fluid is simulated in an energy conserving ensemble; the kinetic temperature is defined as $2 \alpha E_{k} / 3 N$, where $E_{k}$ is the mean kinetic energy of a typical particle in the system and $\alpha$ is the number of number of kinetic degrees of freedom of the system. This differs from $3 N$ due to conservation of angular momentum about the cavity center: $\alpha=3 N-2$ (not $3 N-3$, as would be the case if linear momentum were conserved).

[29] K. Kalyanasundaram, Photochemistry in Microheterogeneous Systems (Academic, New York, 1987); M. Tachiya, in Kinetics of Inhomogeneous Processes, edited by G. R. Freeman (Wiley, New York, 1987).

[30] A solution of the diffusion equation with diffusion constant $\beta$ in a cavity of radius $R$ produces an exact series solution for $\left\langle|\mathbf{r}(t)-\mathbf{r}(0)|^{2}\right\rangle$. Equation (7) then gives

$$
D(t)=\frac{2 R^{2}}{t} \frac{\frac{6}{5}-12 \sum_{\kappa} \frac{e^{-} \beta \kappa^{2} t}{(\kappa R)^{2}\left[(\kappa R)^{2}-2\right]}}{12 \beta \sum_{\kappa} \frac{e^{-} \beta \kappa^{2} t}{\left[(\kappa R)^{2}-2\right]}} .
$$

Summations are over all eigenvalues $\kappa$ such that

$$
j_{1}^{\prime}(\kappa R)=0
$$

where $j_{1}$ is the spherical Bessel function of order unity. [31] G. Vinyard, Phys. Rev. 110, 999 (1958). 